\title{
A new problem arising after the cesarean, cesarean scar defect (isthmocele) a case report
}

\begin{abstract}
Backgraund: Cesarean rates continue to increase all over the world. This has led to a particularly rapid increase in placenta-induced complications. A definition has emerged which is now called the new name istmosel, also called cesarean scar defect. Globally, the frequency is between $6.2 \%$ and $36 \%$. Scar tissue in the old cesarean section causes problems such as menometrorrhagia, staining and infertility in patients. There are laparoscopic and hysteroscopic treatment approaches. Since there is a new terminology, there is no comprehensive publication in the literature to provide a general view on diagnosis and treatment. The primary treatment approach we offer in this case is laparoscopic surgery.
\end{abstract}

Case presentaion: A 37-year-old patient with gravida 2, parity 2, two previous cesarean section stories had complaints of continuing intermenstrual bleeding for 2 years. When the patient's examination and radiological evaluation is done, there was fluid accumulation in the old cesarean section.In the ultrasonographic examination; a dome-shaped fluid collection of about $2.3 \times 1.4 \mathrm{~cm}$ was observed in the uterine lower segment anterior Wall. As a result, laparoscopic approach was decided for istmosel treatment.

Conclusion: There is no consensus in the literature as to what type of suture or energy modality should be used to repair the isthmocele. In addition, there is no consensus on the superiority of double-layer suturing and superiority of the laparoscopic or hysteroscopic approach in cesarean scar repair

Keywords: Cesarean scar defect, İsthmocele, Abnormal uterine bleeding
Volume 7 Issue 5 - 2017

Hakan Nazik,' Evsen Nazik²

'Department of Obstetrics and Gynecology, Health Sciences University, Turkey

2Department of Obstetrics and Gynecology, ?ukurova University, Turkey

Correspondence: Hakan Nazik, Associate Professor, Department of Obstetrics and Gynecology, Health Sciences University, Turkey, Huzurevleri mh. 7724I sk. Bahçe Apt K:7/I3, 01360, Adana,Turkey, Tel 90542686 | I20, Fax 903222472654 , Email drhakannazik@gmail.com

Received: March 17, 2017 | Published: August II, 2017

\section{Introduction}

Cesarean rates continue to increase all over the world.Obstetric complications showed a similar increase with increasing cesarean section rate.With the many obstetric complications of increased cesarean rates, isthmocele terminology as also defined cesarean scar defecthas begun to used in the current literature. Isthmocele is a defect that appears like a dome or pocket that is localized on the old cesarean scar. The global frequency is observed between $6.2 \%$ and $36 \%$, with an average rate of $21.1 \% .^{1}$ This defect including mucus, old blood, debris and inflammatory cells may be responsible for clinical symptoms such as premenstrual bleeding, dysmenorrhea, dyspareunia and infertility. The most reported symptoms about isthmocele are postmenstrual spotting and infertility. The main risk factors are uterine incision closure techniques, suture materials, transverse lower uterine incision and wound healing. ${ }^{2}$ The main reason for the increase in all these risk factors is increased cesarean rates. According to OECD data by 2015, vaginal birth rates are $72.4 \%$ on average, whereas in Turkey this rate is $47 \%$. Several techniques have been described for isthmocele repair These are combined laparoscopic-vaginal and complete vaginal approaches. Laparoscopic excision of the fibrotic tissue at the edges of the caesarean scar or hysteroscopic resection of the fibrotic tissue. The choice of treatment approach should be based on whether the patient has a complaint, the thickness of the myometrium over the cesarean scar defect and the size of the defect. ${ }^{3}$ Today, there is no comprehensive scientific work to provide sufficient information about the ideal approach. In this case report, we described the laparoscopic approach to a patient with abnormal uterine bleeding diagnosed with isthmocele in the light of current literature.

\section{Case report}

A 37-year-old patient with gravida 2, parity 2, two previous cesarean section stories had complaints of continuing intermenstrual bleeding for 2 years. The last cesarean had been 2 years ago. In the history of the patient, after the second cesarean, the patient's vaginal bleeding continued for 2 months in the postpartum period. The patient was examined during this period and in the vaginal ultrasound, $4 \mathrm{~cm}$ servical hematoma was detected on cesarean scar. Therapeutic curettage was applied with a cannula to minimize hematoma. However, despite the intervention, the patient's vaginal spotting bleeding complaints continued for 2 years. Since the patient's complaints did not respond to the medical treatment, she applied to our clinic again.

When the patient's examination and radiological evaluation is done again,there was fluid accumulation in the old cesarean section. In the ultrasonographic examination; a dome-shaped fluid collection of about $2.3 \times 1.4 \mathrm{~cm}$ was observed in the uterine lower segment anterior wall (Figure 1). On the MRI report of the lower abdomen, intensive cystic lesion about $1.9 \mathrm{~cm}$ in diameter with submucosal localization at the level of uterine cervix was observed. Since there is no consensus in the literature on isthmocele therapy, detailed information about treatment options was given to the patient. As a result, laparoscopic approach was decided for istmosel treatment.

\section{Surgical procedure}

To aid in anatomic definition and facilitate bladder dissection, the Clermont Ferrand uterine manipulator (KarlStorz, Gmbh and Co, Tuttlingen, Germany) was used. A main trocar (10 $\mathrm{mm}$ in size) was inserted into the abdominal cavity with the direct insertion techniquefrom the umbilical region after a semicircular incision. After pneumoperitoneum was obtained, 3 trocars $(5 \mathrm{~mm}$ in size, from the right and left inguinal regions and left upper abdominal quadrant) were inserted. 

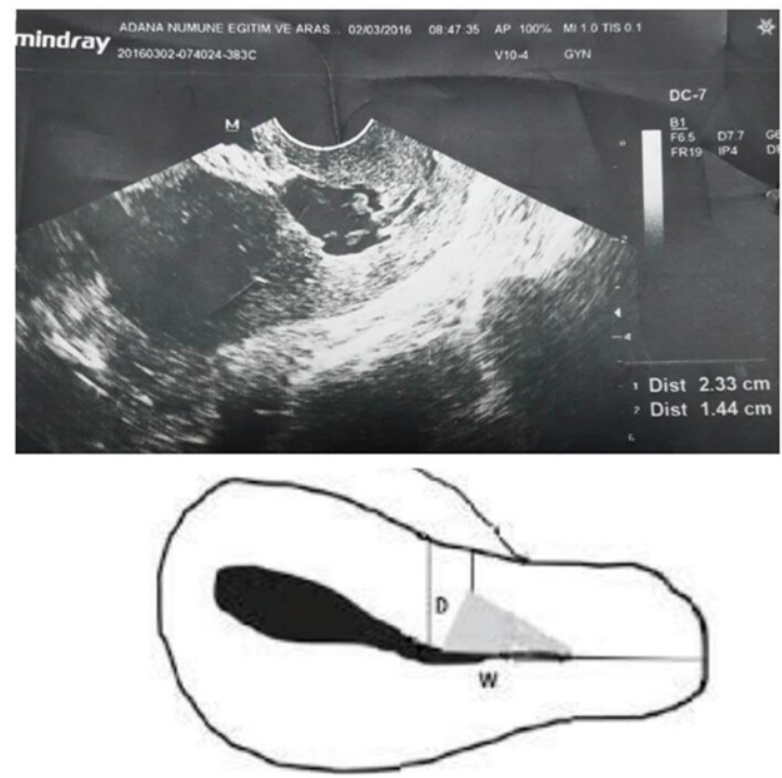

Figure I Ultrasonographic image and diagram of caesarean scar defect "isthmocele".

Uterine fundus, both ovaries and tubules were normalin abdominal exploration (Figure 2A). On the caesarean scar line, a defect was observed that like a dome under the bladder (Figure 2B). Visceral peritoneum covering the isthmus was incised transversally by using bipolar desiccation and scissors, and the bladder was mobilized caudad by blunt and sharp dissections (Figure 2C \& 2D). Caesarean scar was removed all around with the help of harmonic scalpel, (Figure 2E-2G). The scar is removed and the blood and mucus that has accumulated in the cervix is released (Figure 2F). The resulting cervical defect was repaired with a continuous polyglactin $2 / 0$ suture (Figure 2H-2J). Pathologic examination was reported as areas of myocollagenized stromal vein sections and lymphocytic inflammatory cell infiltration areas in adipose tissue.About 2 months after the operation, the patient's complaints improved.

\section{Discussion}

Isthmocele, as in our case, can lead to abnormal uterine bleeding and spotting. Due to the functional endometrium and poor scar contractility, the deteriorating drainage leads to blood clotting in this pocket. This can result in intermenstrual bleeding and pelvic pain. Suppression of menstruation or surgical repair with medical therapy can improve this symptoms. ${ }^{4}$

The cesarean scar defect called as isthmocele, two basic features have been considered in physiopathology. The first of these; the inadequate healing of the caesarean suture line, and the second one is the thickness of myometrium in this area. It has been shown that myometrial thickness can not be increased by hysteroscopic approach in the recent publications in the literature. ${ }^{5}$

In a controlled, randomized study, it was shown that the ratio of istmosel was lower, in patients with endometrial layer full-sutured. ${ }^{6}$ In another prospective cohort study, when the group in which the myometrium was double-ply sutured and were compared the singleply sutured group, less isthmocele was seen in the double-sutured group.

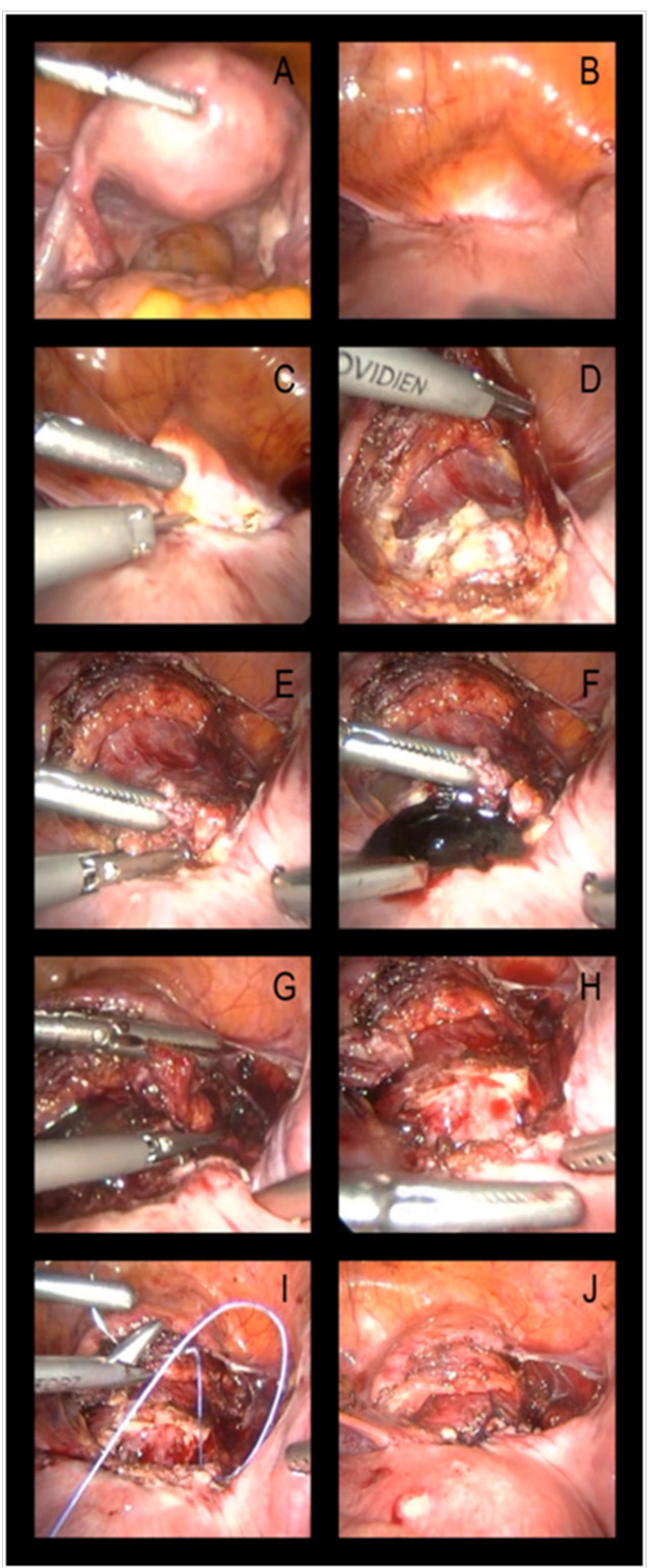

Figure 2 Laparoscopic excision and repair of the cesarean scar defect. (A) Uterine fundus, both ovaries and tubules were normalin abdominal exploration. (B) Laparoscopic view of defect outpouching in lower uterine segment. (C,D) Visceral peritoneum covering the isthmus was incised transversally. (E-G) Caesarean scar was removed all around with the help of harmonic scalpel. $(\mathrm{H}-\mathrm{J})$ The resulting cervical defect was repaired with a continuous polyglactin $2 / 0$ suture. 
In a study investigating the clinical necessity and diagnostic correlation of transvaginal ultrasonography and hysteroscopy in 32 infertile patients, the difference between sonography and hysteroscopic follow-up to confirm the diagnosis was investigated. According to the study result, there was a complete correlation between hysteroscopy and investigation of fluid collection on cesarean scar with transvaginal ultrasonography. ${ }^{8}$

In laparoscopy, different methods have been proposed to determine the location of the istocele. Klemm and colleagues suggested that transvaginal sonography could be performed under laparoscopic vision if the scar piercing can not be immediately seen after cutting the bladder peritoneum and dissection of the uterovascular layer. ${ }^{9}$ In our case, we used uterine manipulator porcelain to distinguish the bladder border.lthough literature has been recently described in terms of different terminologies such as uterine niche, pouch, cesarean scar in cases of infertility, in fact the presence of isthmocele has begun to become a subject of attention in the secondary infertile cases with previous cesarean section. Primer aim is to decrease the rates of cesarean, but in cases where cesarean is inevitable, randomized controlled studies with large case series are needed to investigate the variables such as uterine incision closure techniques, suture materials to remove the factors that cause isthmocele formation.

\section{Conclusion}

There is no consensus in the literature as to what type of suture or energy modality should be used to repair the isthmocele. In addition, there is no consensus on the superiority of double-layer suturing and superiority of the laparoscopic or hysteroscopic approach in cesarean scar repair. Bladder flap creation is a very difficult process in isthmocele cases. For this reason, the surgeon should use a method that is experienced. Otherwise bladder injury is an unavoidable complication. Controlled clinical trials involving large case series are very poor in the literature on isthmocele repair. In our world where the rates of caesarean are increasing, we think that it will be possible to carry out extensive studies on this subject in the future.

\section{Acknowledgements}

We would like to thank İlker Gül for his kind help on graphics and design.

\section{Conflicts of Interest}

Authors declare that this article has not been supported by any organization.

\section{References}

1. Nezhat C, Soliemannjad R, Razavi GM, et al. Cesarean scar defect: what is it and how should it be treated? OBG Management. 2016;28(4):32-53.

2. Raimondo G, Grifone G, Raimondo D, et al. Hysteroscopic treatment of symptomatic cesarean-induced isthmocele: a prospective study. $J$ Minim Invasive Gynecol. 2015;22(2):297-301.

3. Donnez O, Donnez J, Orellana R, et al. Gynecologic and obstetric outcomes after laparoscopic repair of a cesarean scar defect in a series of 38 women. Ferti Steril. 2017107(1):289e2-296.e2.

4. Armstrong V, Hansen WF, Van Voorhis BJ, et al. Detection of Cesarean scars by transvaginal ultrasound. Obstet Gynecol. 2003;101(1):61-65.

5. Api M, Boza A, Gorgen H, et al. Should Cesarean Scar Defect Be Treated Laparoscopically? A Case Report and Review of the Literature. $J$ Minim Invasive Gynecol. 2015;22(7):1145-1152.

6. Yazicioglu F, Gökdogan A, Kelekci S, et al. Incomplete healing of the uterine incision after caesarean section: Is it preventable? Eur J Obstet Gynecol Reprod Biol. 2006;124(1):32-36.

7. Hayakawa H, Itakura A, Mitsui T, et al. Methods for myometrium closure and other factors impacting effects on cesarean section scars of the uterine segment detected by the ultrasonography. Acta Obstet Gynecol Scand. 2006;85(4):429-434.

8. Fabres C, Aviles G, De La Jara C, et al. The cesarean delivery scar pouch: clinical implications an diagnostic correlation between transvaginal sonography and hysteroscopy. J Ultrasound Med. 2003;22(7):695-700.

9. Klemm P, Koehler C, Mangler M, et al. Laparoscopic and vaginal repair of uterine scar dehiscence following cesarean section as detected by ultrasound. J Perinat Med. 2005;33(4):324-331. 